\title{
Assistive Navigation Device for Visually Impaired-A Study on Reaction Time to Tactile Modality Stimuli
}

\author{
Jing Yu, Knut Moeller \\ Institute of Technical Medicine, Furtwangen University, Furtwangen im Schwarzwald, Germany \\ Email: jing.yu@hs-furtwangen.de
}

Received May 2013

\begin{abstract}
A tactile system to support severe visually-impaired or blind people in the world for their orientation and navigation had been developed. To optimize the design, some parameters of tactile display device were evaluated. In the present paper, we focused on the reaction time to tactile stimuli. In the test, the stimuli were produced through a vibration belt that was worn around the participants' waist. In the choice reaction time task, the participants had to click corresponding arrow keys according to the location of a tactile signal. The findings of this study provided a reference of the reaction time range, so as to design a more effective and safe tactile navigation system.
\end{abstract}

Keywords: Tactile Display; Reaction Time; Skin Receptor; Assistive Technology; Vibration Belt

\section{Introduction}

Globally, an estimated 285 million people are visually impaired, of whom 39 million are blind and 246 million have low vision [1]. Over the last decades, all kinds of electronic assistive devices have been proposed for safe and independent mobility of the visually impaired. The assistive systems scan the environment (using different technologies, such as sonar, 3D camera [2]) and display the information to other senses. The human-machineinterfaces nowadays not only routinely utilize the auditory stimuli modality, but also increase the use of tactile modality.

Auditory display is not widely accepted among blind people, since the sound from voice synthesizers would conflict with other sounds in the surrounding environment [3]. The tactile display is more desirable to transmit the visual information for several reasons. The surface area of the skin is very large. In addition, tactile displays provide information in an unobtrusive way [4], and so this kind of system is suited for users working with other tasks. Therefore, in our system we chose the sense of touch to reduce visual information deficiencies for blind people.

Various forms of assistive devices that can be worn on different parts of the torso have been developed, such as finger [5], wrist, forearm [6], tongue [7], feet [8], chest, belly [9-11] and head [3]. Considering the sensitivity and adaptability, we developed a belt-type device.

For safety in the navigation task, it is important to know how far ahead of time a tactile signal should be sent to the visually impaired people at least. That time depends on reaction time. Reaction time refers to the interval of time between the application of a stimulus and detection of a response.

Reaction time tasks can be classified according to the number of diverse stimuli in a task that need to be responded. When the number of stimuli is equal to one, this kind of reaction time task is called the simple reaction time task. Simple reaction time task is an issue when only one particular stimulus can occur or the same response is always required. If the number of stimuli is higher than one, it is defined as choice reaction time task. This kind of task requires a particular response for each stimulus [12].

A number of studies have been conducted to analyze the influence of different factors on human response time such as stimulus modality, stimulus intensity, gender, stimulus-response alternatives, and stimulus location [13, 14].

In this paper, a choice reaction test was designed to investigate reaction time to the tactile stimuli from the vibration belt.

\section{Methods}

\subsection{Participants}

A total of 21 participants aged between 19 and 35 years, voluntarily took part in this study. All of them were in good visual, auditory, and physical condition at the time of the study. 


\subsection{Hardware}

The tactile signals were issued from vibration motors which were integrated into a simple belt (Figure 1) worn around the waist. The number and position of the motors could be adjusted as required.

These vibration motors (model number LA4-432A) (Figure 2 Left) for the stimulation of the skin receptors are commercially available by the Nidec Copal Company (USA) and are mainly used in mobile phones. The size is $16 \times 5 \times 6 \mathrm{~mm}$. The rated voltage of them is $3 \mathrm{~V}$, the rated speed $9500 \mathrm{rpm}$ and the rated current $66 \mathrm{~A}$. To assure smooth revolutions, each vibration was packed in a small plastic tube.

In the test, the vibration motors are controlled with a NI-USB card (Figure 2 Right) from the National Instruments Corporation (USA). The NI-USB card is connected to the PC with a USB cable. The power supply for the motors was carried out via the voltage output pins of the NI-USB card. An application program prepared with C\# was used to generate tactile stimuli and to capture the participants' responses. A key board was placed at a convenient location at the front of the participants and was used to click the corresponding arrow key.

\subsection{Procedure}

First, the participants were briefed with the objectives

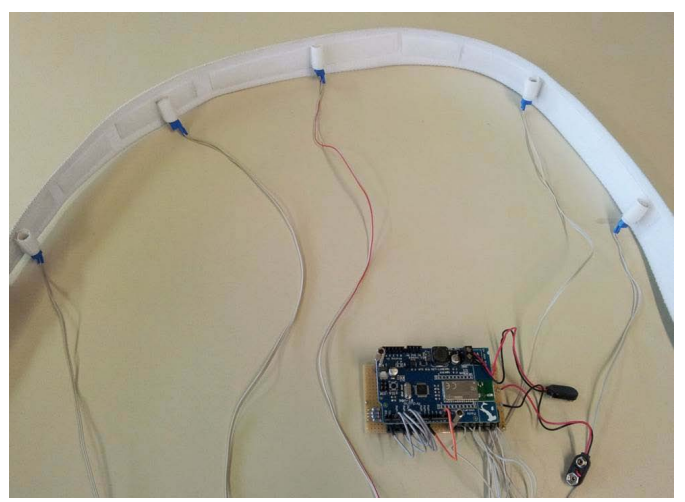

Figure 1. Prototype of the vibration belt, number and position of the motors are adjustable.
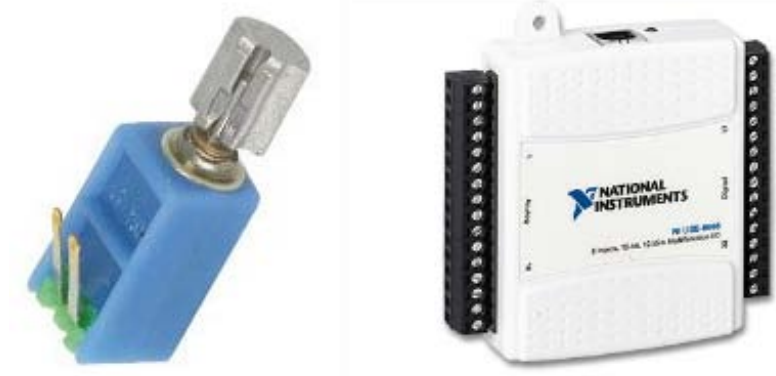

Figure 2. Left vibration motor and right NI-USB card. and procedure of the study. Then they had to wear the vibration belt with four motors which represented four directions by their locations. The one near the navel represented front, the one near the spine represented back, the one on the left side represented left and the one on the right side right. Before the test, the motors were controlled to vibrate one by one to make sure the participants could feel the vibration.

The test was a choice reaction time task. The motors would vibrate 30 times randomly in all different directions. Each vibration pulse lasted 200 ms. Once the participants detected a vibration, they pressed the corresponding arrow key as quickly as possible. After the computer detected the click, another vibration signal would be generated.

\section{Results}

A total of 630 responses (21 participants $\times 30$ pulses) were recorded in the tactile stimuli test. Descriptive statistics of reaction time were assessed.

\subsection{Average Reaction Time and Standard Deviation}

The average reaction time for tactile stimuli was 489 ms with standard deviation of $142 \mathrm{~ms}$. The average reaction time and standard deviation for each participant were plotted in Figure 3 which was sorted by increasing average reaction time of each participant.

\subsection{Position of Vibrators}

To evaluate the influence of vibration location, the aver-

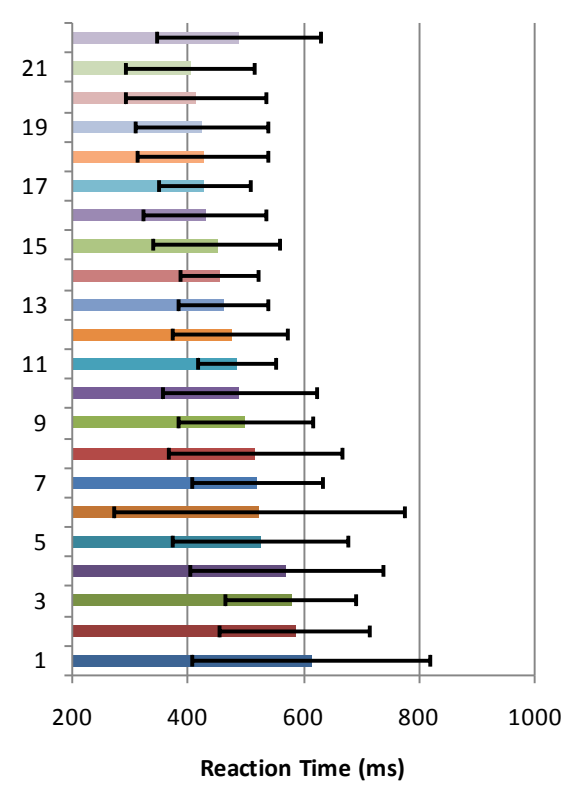

Figure 3. The average reaction time and standard deviation for each participant. 
age reaction time and deviation were plotted in Figure 4.

\subsection{Correct and Wrong Response}

21 individuals participated in the study; 14 of them made wrong responses. 630 responses were obtained, out of which 38 were false reactions. The average reaction time of correct responses was $490 \mathrm{~ms}$, and the average of wrong responses was $465 \mathrm{~ms}$.

\section{Discussion}

This study was about reaction time in response to the tactile stimuli from a vibration belt. Even for the young age group, the range of reaction time is very wide (Figure 3). Our design of assistive navigation device aimed to make the life of visually impaired people more convenient and independent. Since security is a major factor of such a device, it is important to know how far ahead of time a tactile signal should be sent at least to allow appropriate actions to be taken by the visually impaired. According to the results, the tactile signal should be individually adapted and sent between 600 and $900 \mathrm{~ms}$ ahead.

According to the data from different location (Figure 4), the reaction time of left and right is shorter than the front and back and it might be hypothesized that reaction time is location specific. Because of the design of key board, people would like to use index finger to press left arrow, fourth finger to press right and middle finger to press up and down. So reaction time might involve longer decision time to select and press the appropriate up or down arrow keys. Further experiments need to be performed to come to a conclusion if left and right sides are more sensitive than the front and back.

On the average, the reaction time of false responses is shorter than the correct ones. Since the data of wrong responses are not enough for a statistical analysis and to prove significance of differences, further evaluations will be performed in the next experimental steps.

For further work, some other factors should be taken into account too. First, age: the finding that the reaction time increases with the increase of age has been reported by Ashoke et al. [15]. With a group of subjects of 5 to 70

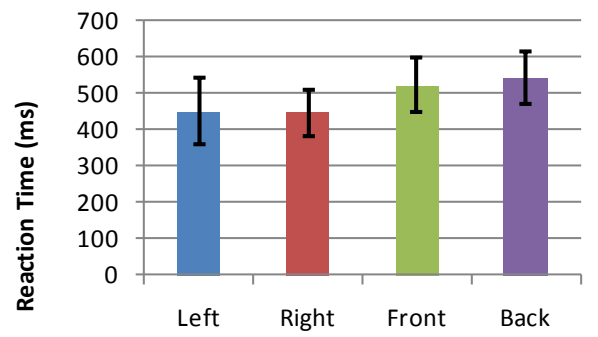

Figure 4. The average reaction time and deviation of different locations. years of age, they found that the response was faster with an increase of age up to 21 - 25 years and then responsetimes gradually increased with age. Second, the complexity of response: Kamitani et al. [16] found that the response on choice reaction time task was significantly longer than simple reaction time task. According to this study, the possible explanation of such finding was that choice reaction task required not only stimulus perception and execution of the response but also decisionmaking processes.

Overall, even the combination of the effects of different factors should be considered in future study. The present finding is still a useful reference to design a safe tactile navigation system for visually impaired.

\section{Acknowledgements}

This work was supported by the Bundesministerium für Bildung und Forschung (BMBF), IH-2 iVIEW, FKZ 13EZ1129A.

\section{REFERENCES}

[1] World Health Organization, "Visual Impairment and Blindness," Fact Sheet No. 282, 2012. http://www.who.int/mediacentre/factsheets/fs282/en/

[2] K. Möller, J. Möller, K. O. Arras, M. Bach, S. Schumann and J. Guttmann, "Enhanced Perception for Visually Impaired People Evaluated in a Real Time Setting," World Congress on Medical Physics and Biomedical Engineering, Vol. 25, 2009, pp. 283-286.

[3] R. Velázquez, "Wearable and Autonomous Biomedical Devices and Systems for Smart Environment: Issues and Characterization,” In: A. Lay-Ekuakille and S. C. Mukhopadhyay, Eds., LNEE 75, Springer, Berlin, 2010, pp. 331-349.

[4] F. A. Geldard, "Some Neglected Possibilities of Communication,” Science, Vol. 131, 1960, pp. 1583-1588. http://dx.doi.org/10.1126/science.131.3413.1583

[5] I. M. Koo, K. Jung, J. C. Koo, J. Nam, Y. K. Lee and H. R. Choi, "Development of Soft-Actuator-Based Wearable Tactile Display,” IEEE Transactions on Robotics, Vol. 24, No. 3, 2008, pp. 549-558. http://dx.doi.org/10.1109/TRO.2008.921561

[6] G. Ng, P. Barralon, G. Dumont, S. Schwarz and J. Ansermino, "Optimizing the Tactile Display of Physiological Information: Vibro-Tactile vs. Electro-tactile stimulation, and Forearm or Wrist Location,” Annual International Conference of the IEEE Engineering in Medicine and Biology Society, Lyon, 22-26 August 2007, pp. 42024205.

[7] M. Ptito, S. Moesgaard, A. Gjedde and R. Kupers, "CrossModal Plasticity Revealed by Electrotactile Stimulation of the Tongue in the Congenitally Blind,” Brain, Vol. 128, No. 3, 2005, pp. 606-614. http://dx.doi.org/10.1093/brain/awh380

[8] R. Velazquez, O. Bazan and M. Magaña, “A Shoe- Inte- 
grated Tactile Display for Directional Navigation,” Proceedings of IEEE/RSJ International Conference on Intelligent Robots and Systems, St. Louis, 10-15 October 2009, pp. 1235-1240.

[9] J. Borenstein, "The NavBelt-A Computerized MultiSensor Travel Aid for Active Guidance of the Blind," Proceedings of the CSUN's Fifth Annual Conference on Technology and Persons with Disabilities, Los Angeles, 21-24 March 1990, pp. 107-116.

[10] K. Tsukada and M. Yasumrua, "ActiveBelt: Belt-Type Wearable Tactile Display for Directional Navigation,” Proceedings of UbiComp2004, Nottingham, 7-10 September 2004, pp. 384-399.

[11] S. Nagel, C. Carl, T. Kringe, R. Märtin and P. König, "Beyond Sensory Substitution-Learning the Sixth Sense,” Neural Engineering, Vol. 2, 2005, pp. R13-R26. http://dx.doi.org/10.1088/1741-2560/2/4/R02

[12] A. W. Y. Ng and A. H. S. Chan, "Finger Response Times to Visual, Auditory and Tactile Modality Stimuli," Proceedings of the International MultiConference of Engineers, Hong Kong, 14-16 March 2012.

[13] L. R. Duncan and V. S. Thomas, "Response Times: Their Role in Inferring Elementary Mental Organization,” Oxford University Press, New York, 1991.

[14] E. R. Hoffmann and J. T. A. Lim, "Concurrent ManualDecision Tasks,” Ergonomics, Vol. 40, 1997, pp. 293-318. http://dx.doi.org/10.1080/001401397188161

[15] B. Ashoke, D. Shikha and B. Sudarsan, "Reaction Time with Respect to the Nature of Stimulus and Age of Male Subjects," Sport and Health Research, Vol. 2, No. 1, 2010, pp. 35-40.

[16] T. Kamitani, Y. Kuroiwa, M. Li, T. Ikegami and S. Matsubara, "Relationship between Cerebellar Size and Variation of Reaction Time during a Visual Cognitive Task in Normal Subjects,” Neurology, Vol. 250, 2003, pp. 10011003. http://dx.doi.org/10.1007/s00415-003-1149-2 